\title{
Method of Separation of Incidental Acoustic Field on Cylindrical Shell by Vector Processing
}

\author{
Baoshan Yang $\mathbb{D},{ }^{1}$ Ma Zhongcheng $\mathbb{D}^{1,2}$ and Lingguo Zhu $\mathbb{D i D}^{1,3}$ \\ ${ }^{1}$ Dalian Scientific Test and Control Technology Institute, Dalian 116013, China \\ ${ }^{2}$ Science and Technology on Underwater Test and Control Laboratory, Dalian 116013, China \\ ${ }^{3}$ Harbin Engineering University, Harbin 150001, China \\ Correspondence should be addressed to Ma Zhongcheng; 13704261100@163.com
}

Received 11 February 2020; Accepted 11 July 2020; Published 12 August 2020

Academic Editor: Francesco Franco

Copyright ( $\odot 2020$ Baoshan Yang et al. This is an open access article distributed under the Creative Commons Attribution License, which permits unrestricted use, distribution, and reproduction in any medium, provided the original work is properly cited.

\begin{abstract}
A theoretical and experimental study on the separation method of the incident sound field based on a small-scale vector sensor is proposed in this study, with the aim of resolving the problem of separation and acquisition of an incident sound field under the interference of near-field sound scattering from a cylindrical shell in water. The method of identifying and separating sound waves obtained under plane wave conditions is extended to complex sound-field conditions. Simulation and experimental results show that the vector separation method can greatly reduce the sound pressure amplitude and the phase deviation of the incident sound field, which is affected by near-field scattering from the cylindrical surface. The separation accuracy is related to the deviation angle and the distance from the target surface. The maximum deviation of the pressure amplitude is less than $1 \mathrm{~dB}$, and the phase deviation is less than $3^{\circ}$. This method can effectively suppress the near-field scattering of the cylindrical shell and improve the separation accuracy of the incident sound field. The research results have reference value for a range of practical engineering applications.
\end{abstract}

\section{Introduction}

The cylindrical shell structure is the most common form of an underwater sports platform. Due to the influence of scattering near the shell surface, the incident acoustic signal received by a sensor on the shell surface in the sound field will display severe distortion. Incident sound field separation is used to study the suppression of near-field scattering on the surface of the shell and accurately obtain the amplitude and phase information of the incident sound field. Active acoustic feature control $[1,2]$, accurate response measurement $[3,4]$, underwater acoustics for underwater target positioning, and other factors are instructive for guiding the sound field separation process $[5,6]$.

At present, the near-field acoustic holography (NAH) method is mainly used to examine the distribution of the sound field and perform the separation of the incident sound field on the surface of the cylindrical shell [7]. Reference [8] is based on a two-sided NAH technology. When the incident sound field and the axis of the holographic plane are not parallel, the separation of the incident sound field and the scattered sound field was achieved Based on the boundary element method (BEM), the separation method of incident and scattered acoustic fields is studied $[9,10]$. For the incident and scattered sound fields, wave-field extrapolation theory in the wavenumber domain and the scalar superposition principle of sound pressure was used to establish a dual-holographic surface sound field separation method and a single-layer nearfield, based on the principle of vector velocity superposition $[11,12]$. For the holographic sound-field separation method, Li et al. established the separation formula of incident and scattered sound fields in spherical coordinates in the wavenumber domain through the sound pressure decomposition of two concentric spheres [13]. This overcomes the ill-posed nature of the inverse problem of acoustics. However, these methods need to build a near-field acoustic holographic array with a certain scale and density, which is difficult to achieve for targets at sea, especially underwater.

Acoustic vector sensors can simultaneously obtain a scalar sound pressure and a three-dimensional particle 


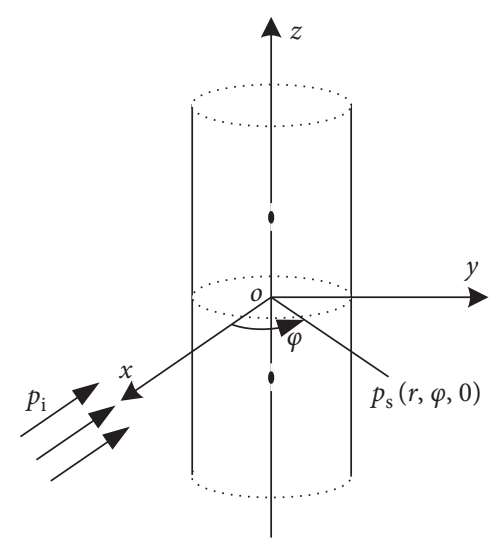

(a)

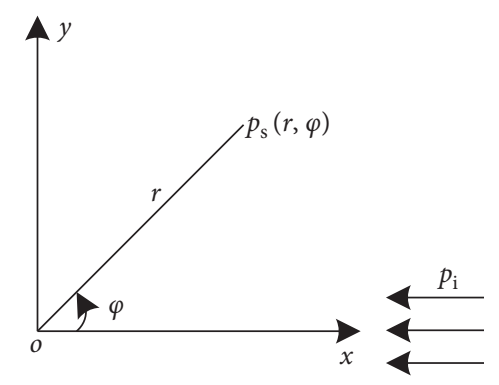

(b)

FIgure 1: Model of the surface acoustic field of an infinitely long rigid cylinder. (a) Three-dimensional. (b) Two-dimensional.

velocity. The combined processing of scalar and vector components has advantages over individual processing. Based on the underwater acoustic buoy in the form of a hollow ball, the distortion of the sound field caused by diffraction on the surface of the underwater acoustic device carrier was studied [14]. Studies on the basis of joint information processing, such as the correlation and directivity of sound pressure and vibration velocity in underwater acoustic fields, have also been conducted. These works identified the coherent interference synthesis problem of energy flow suppression technology [15-17]. The effect of scattering by a spherical shell baffle on vector directivity and far-field incident acoustic signal measurement has also been theoretically and experimentally studied [18]. The abovementioned literature focuses on the surface of spherical structures. The influence of the sound-field distribution and its scattering on the incident sound field has not been studied for the common distribution of the sound field on the surface of a cylindrical shell, including the precise separation of the incident sound field.

The acoustic field on the surface of a cylindrical shell is the superposition of the far-field incident wave and the surface near-field scattered wave. Based on the distribution of the surface acoustic field of the cylinder, this study proposes a method to separate the incident sound field by combining the processing of the sound pressure and the particle velocity. The obtained sound wave identification and the separation theory are extended to complex soundfield conditions, with the important characteristic of retaining the amplitude and phase information of the sound field. The relationships between the sound-field distribution on the surface of the cylinder and the separation error of the incident sound field with the deviation angle as well as distance are analyzed theoretically and numerically. The separation method of the incident sound field is verified using an actual cylindrical shell model sea test, and the measured separation error is given.

\section{Methods: Sound Field Distribution on Cylinder Surface}

The acoustic field calculation of the near-field of the underwater target mainly uses the classical Rayleigh normal series solution and numerical solution. The normal series solution can be used to obtain the accurate sound-field solutions of several targets with regular geometric shapes. This can deeply reflect the physical mechanisms affecting the sound field and is a test benchmark for other numerical calculation methods. There is no strict analytical solution for the scattered sound field of a finite-length cylindrical shell. Nevertheless, the main source of near-field scattering of the shell is adjacent to the local surface. Therefore, when the wavelength of the sound wave is much smaller than the length of the cylindrical shell and the research object is far away from the end face of a cylindrical shell, the analytical solution of an infinitely long cylinder can be used to analyze the near-field and sound-field distribution law of a finite-length cylindrical shell.

As shown in Figure 1(a), the center of the cylindrical section is used as the origin of the coordinate system, and the incident plane wave is vertically aligned with the infinite rigid cylinder along the $x$-axis. The scattering field is independent of the $z$-direction; it is symmetric with respect to the azimuth angle $\phi$ and is simplified to a two-dimensional problem in the plane $x-y$ as shown in Figure 1(b). The expression of incident sound pressure $\left(p_{i}\right)$ and scattered sound pressure $\left(p_{s}\right)$ is given by the following equation:

$$
\left\{\begin{array}{l}
p_{i}(r, \phi)=2 \sum_{m=0}^{\infty} \zeta_{m} i^{m} J_{m}\left(k_{0} r\right) \cos (m \phi), \\
p_{s}(r, \phi)=\sum_{m=0}^{\infty} A_{m} H_{m}^{(1)}\left(k_{0} r\right) \cos (m \phi),
\end{array}\right.
$$




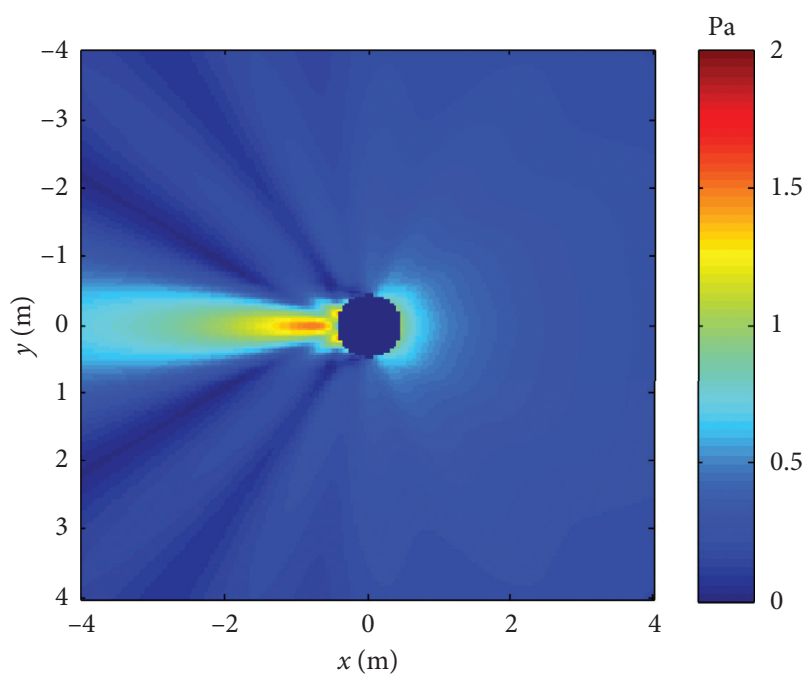

(a)

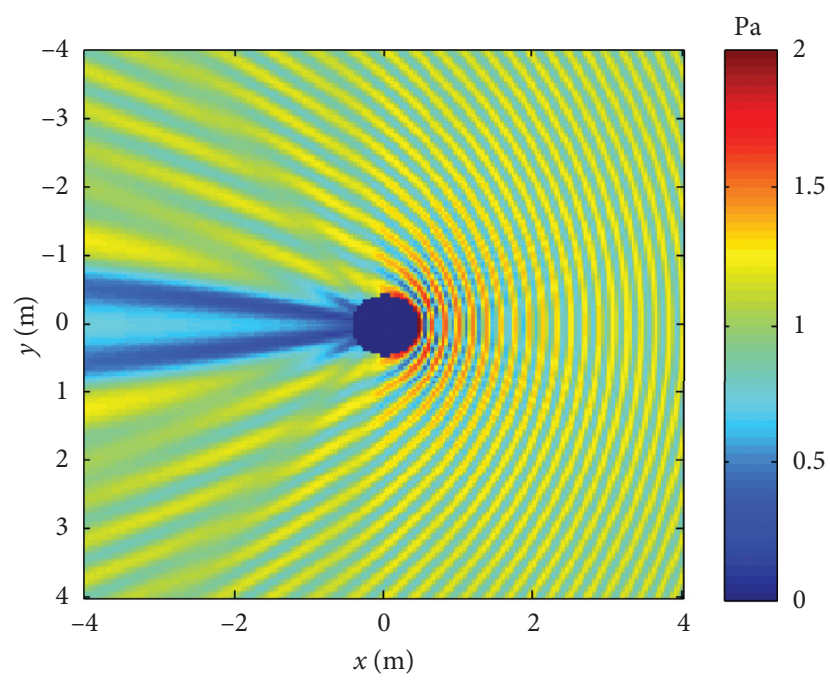

(b)

FIgURe 2: Distribution of the sound field near the cylinder surface for a wave incident from the left. (a) Scattered sound field. (b) Total sound field.

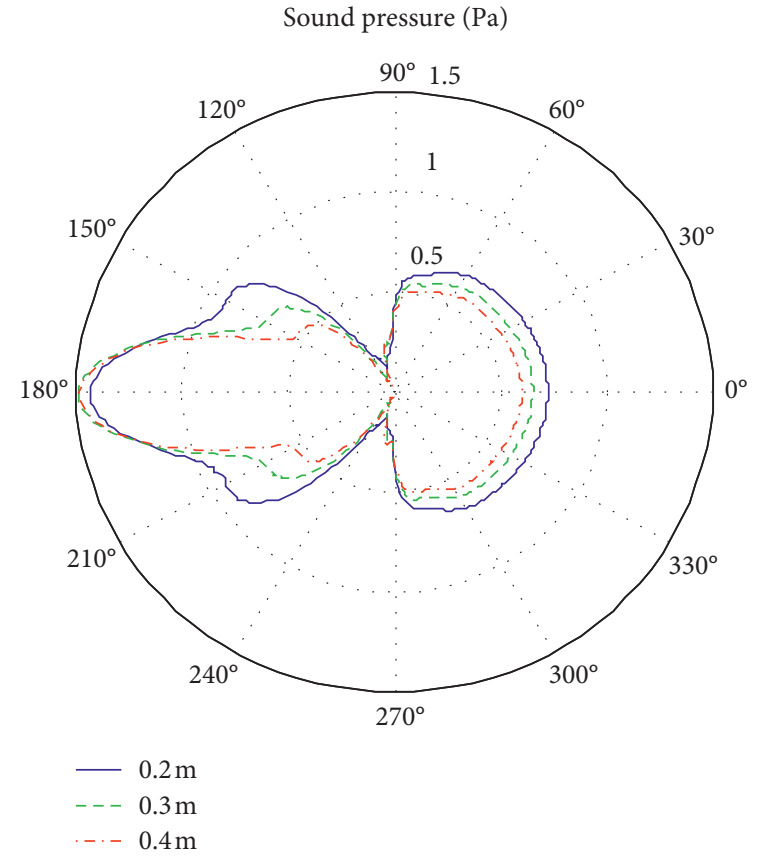

(a)

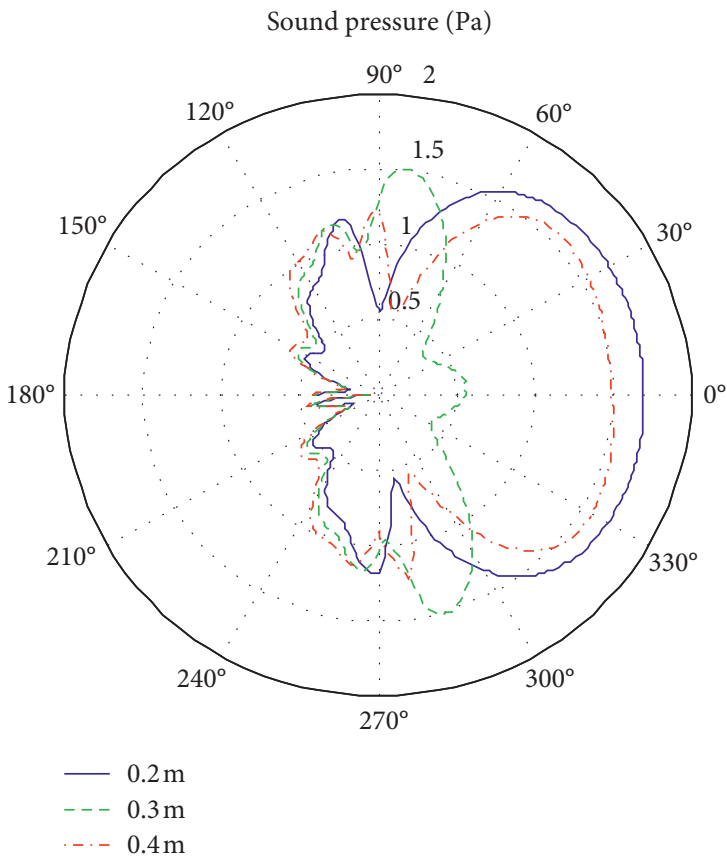

(b)

Figure 3: Azimuth characteristics of the near-field sound field at typical distances. (a) Scattered sound field. (b) Total sound field.

where $\zeta_{m}=\left\{\begin{array}{ll}0.5, & m=0, \\ 1, & m \geq 1\end{array}, \quad k_{0}=\omega / c_{0}, \quad A_{0}=-\left(J_{1}\left(k_{0} a\right)\right) /\right.$ $\left(H_{1}^{(1)}\left(k_{0} a\right)\right), \quad A_{m}=-2 i^{m}\left(\left(J_{m}^{\prime}\left(k_{0} a\right)\right) /\left(H_{m}^{\prime}(1)\left(k_{0} a\right)\right)\right), \quad \omega$ is omega, $c_{0}$ is speed of sound, $k_{0}$ is wavenumber, and $a$ is radius.
The derivatives of the Bessel function and the Hankel function of the first kind are obtained by the recursive formula.

By deriving the potential function corresponding to equation (1), the radial and tangential vibration velocities 


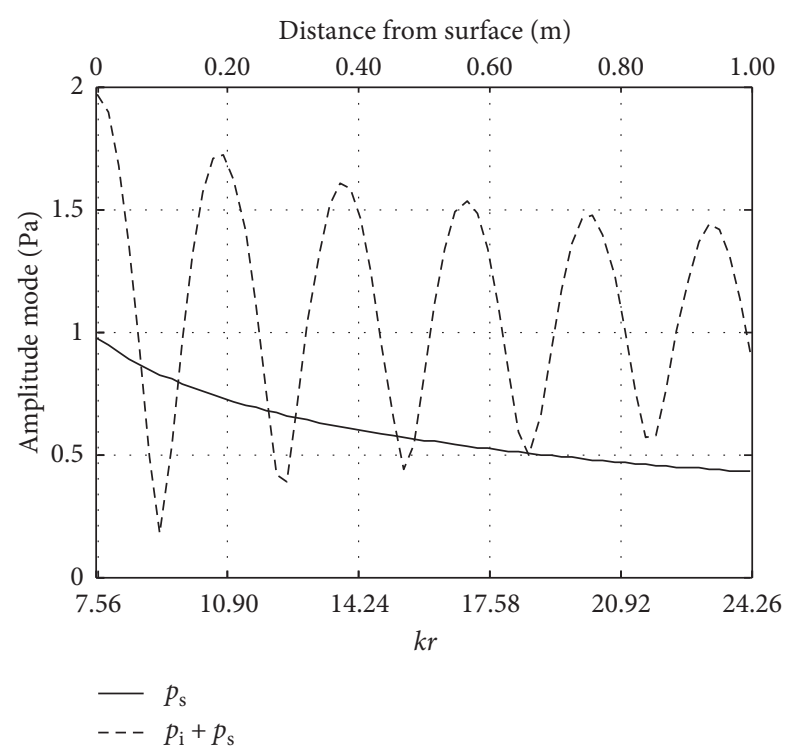

(a)

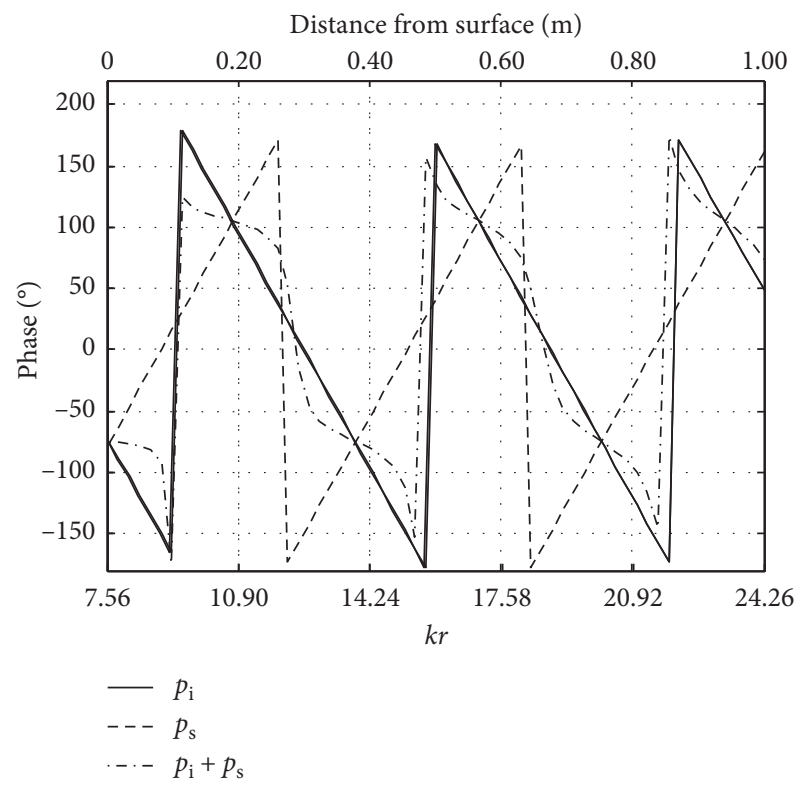

(b)

Figure 4: Distribution characteristics of the sound-field pressure after the cylindrical surface with distance ( $k$ is wavenumber; $r$ is the distance from the geometric center of the target). (a) Amplitude. (b) Phase.

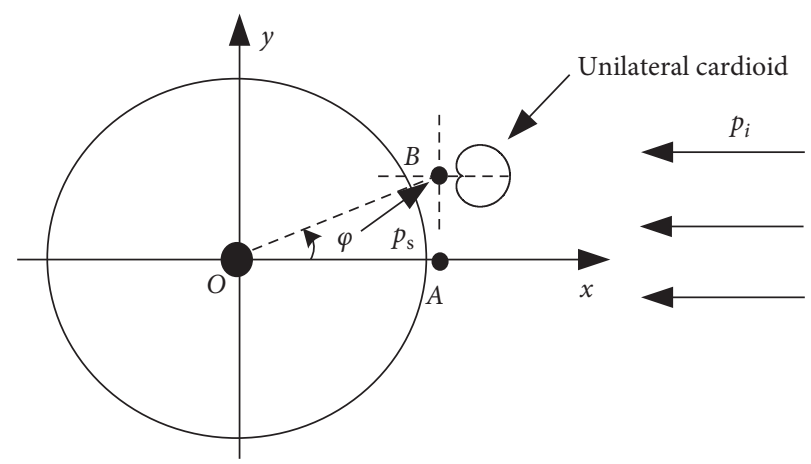

FIGURE 5: Sketch of receiving points near the cylinder surface.

were obtained. From this, the incident $\left(v_{\mathrm{i}}\right)$ and scattered $\left(v_{\mathrm{s}}\right)$

sound velocities in the incoming direction are

$$
\left\{\begin{array}{l}
v_{\mathrm{i} x}(r, \phi)=\frac{1}{i \rho_{0} c_{0}}\left[-J_{1}\left(k_{0} r\right)+2 \sum_{m=1}^{\infty} i^{m} J_{m}^{\prime}\left(k_{0} r\right) \cos (m \phi)\right] \cos (\phi)-2 m \sum_{m=1}^{\infty} i^{m} J_{m}\left(k_{0} r\right) \sin (m \phi) \sin (\phi), \\
v_{\mathrm{s} x}(r, \phi)=\frac{1}{i \rho_{0} c_{0}}\left[-A_{0} H_{1}^{(1)}\left(k_{0} r\right)+\sum_{m=1}^{\infty} A_{m} H_{m}^{(1)}\left(k_{0} r\right) \cos (m \phi)\right] \cos (\phi)-2 m \sum_{m=1}^{\infty} A_{m} H_{m}^{(1)}\left(k_{0} r\right) \sin (m \phi) \sin (\phi) .
\end{array}\right.
$$

The total field of the target surface acoustic pressure and incoming wave velocity is

$$
\left\{\begin{array}{l}
p(r, \phi)=p_{\mathrm{i}}(r, \phi)+p_{\mathrm{s}}(r, \phi) \\
v_{x}(r, \phi)=v_{\mathrm{i} x}(r, \phi)+v_{\mathrm{s} x}(r, \phi)
\end{array}\right.
$$

Considering the physical model geometry parameters and experimental verification frequency in practical applications, the cylinder radius is taken as $0.45 \mathrm{~m}$, and the analysis frequency is selected as $4 \mathrm{kHz}$. The sum of the normal series of finite orders will produce a certain error depending on the number of terms used. However, it is generally considered best practice to take the highest number of terms as $m_{\max } \geq \mathrm{ka}+5$ to guarantee the accuracy of the calculation. In this study, $m_{\max }=30$, the magnitude of 


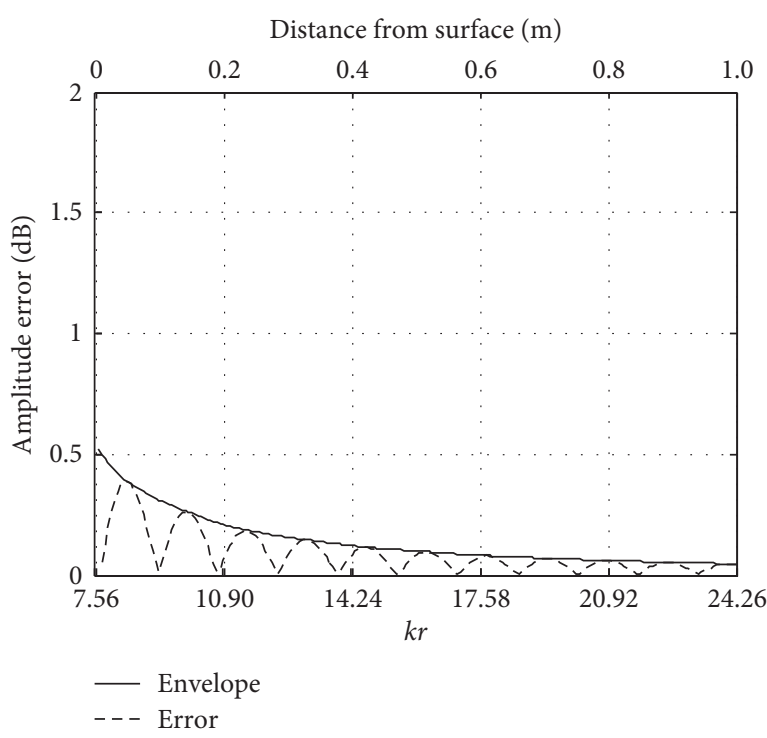

(a)

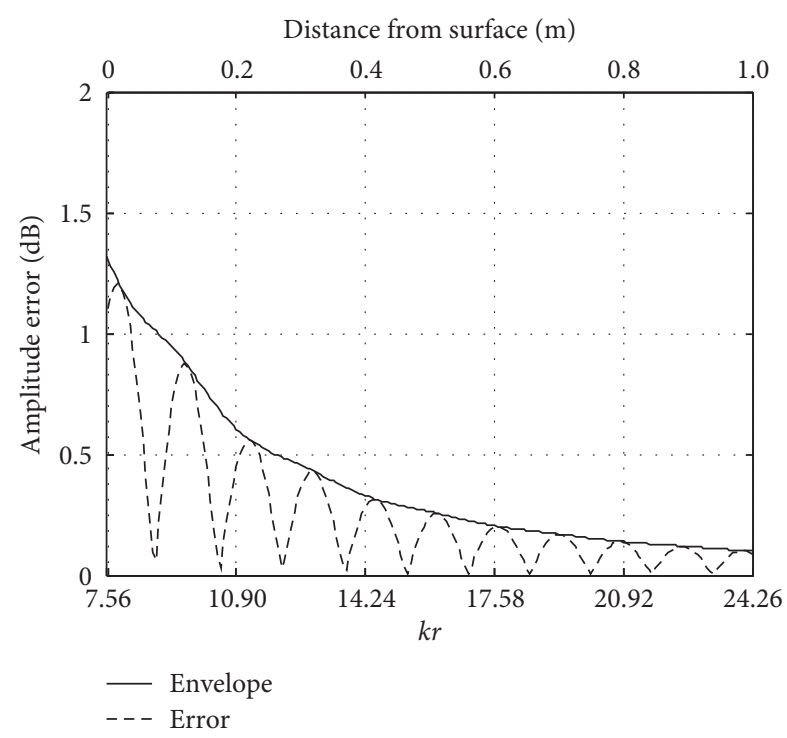

(b)

FIGURE 6: Variation of the separation error of the sound pressure amplitude of the incident sound field with distance. (a) 0 degrees. (b) 30 degrees.

the error in the numerical calculation is less than $5 \%$, and the phase error is less than $2^{\circ}$.

For the distribution of the scattering sound field and total sound field of the cylinder $(a=0.45 \mathrm{~m}, \mathrm{ka}=7)$, the difference between the forward and reverse sound fields is significant, as shown in Figure 2. For the case shown, where the wavelength is comparable to the target radius, the forward scattering sound field (negative $x$-axis directions) is far greater than the reflected sound field (positive $x$-axis directions). However, due to the spatial amplitude, and especially the phase difference between the incident wave and the scattered wave, the total sound pressure in the forward space (negative $x$-axis directions) is far smaller than the total sound pressure in the back space (positive $x$ axis directions). Around the cylinder, the total sound field shows regular fluctuations between the strong and weak fields emitted by the cylinder, thereby forming interference fringes; behind the cylinder (negative $x$-axis value), the total sound field weakens and forms a shadow area.

Since the sensor is generally installed near the surface of the cylinder, the positions of $0.2 \mathrm{~m}, 0.3 \mathrm{~m}$, and $0.4 \mathrm{~m}$ on the surface of the cylinder are selected, and the quantitative change of the sound field is observed, as shown in Figure 3. The extremely near-field forward and backward scattering sound fields are monotonically attenuated. However, after superimposition with the incident waves, the total sound pressure in the back space (positive $x$-axis directions) alternates with strong fluctuations.

We focus on the sound-field distribution on the side of the incident sound field of the cylinder. From the distribution of sound pressure and phase with distance, we can clearly observe and understand the change and superposition of the three sound fields (incident $p_{\mathrm{i}}$, scattered $p_{\mathrm{s}}$, and the superposition $\left.p_{\mathrm{s}}+p_{\mathrm{i}}\right)$. Figure 4 shows the change of amplitude and phase of the three sound fields with horizontal distance (return to cylindrical surface) and $k r$ (dimensionless distance, factor of deduction frequency) in one side of the incident sound field (positive $x$-axis directions). The near-field backscattered sound field is monotonically attenuated. When the incident wave and the scattered wave are in phase, the total sound pressure is the sum of the two, and the total sound field has a peak maximum value (when they are $180^{\circ}$ out of phase, the total sound pressure is the difference between the two, and there is a trough minimum value). Because the amplitude of the scattered sound is smaller than that of the incident sound, the phase position of the total sound field is mainly controlled by the incident sound field. However, the maximum phase fluctuation of the sound field is about $50^{\circ}$ due to the influence of the scattered sound. With the increase in distance from the target, the amplitude and phase difference between the total sound field and the incident sound field gradually decreased. When $k r$ is approximately 17.6 (i.e., $0.6 \mathrm{~m}$ from the cylinder surface), the phase fluctuation value decreased to $20^{\circ}$. Nevertheless, the amplitude fluctuation still exceeded $50 \%$.

2.1. Methods: Incident Sound Field Separation on the Surface of a Cylinder. According to the distribution of the incident and backscattered sound fields around the cylinder surface, the coordinate system shown in Figure 5 was established. The plane wave was incident in the $x$-axis direction towards the origin, the sound pressure and vibration velocity are $180^{\circ}$ out of phase, and the horizontal azimuth angle is measured relative to the $x$-axis. Focus was placed on the area on one side of the incident sound field. The receiver was located at 

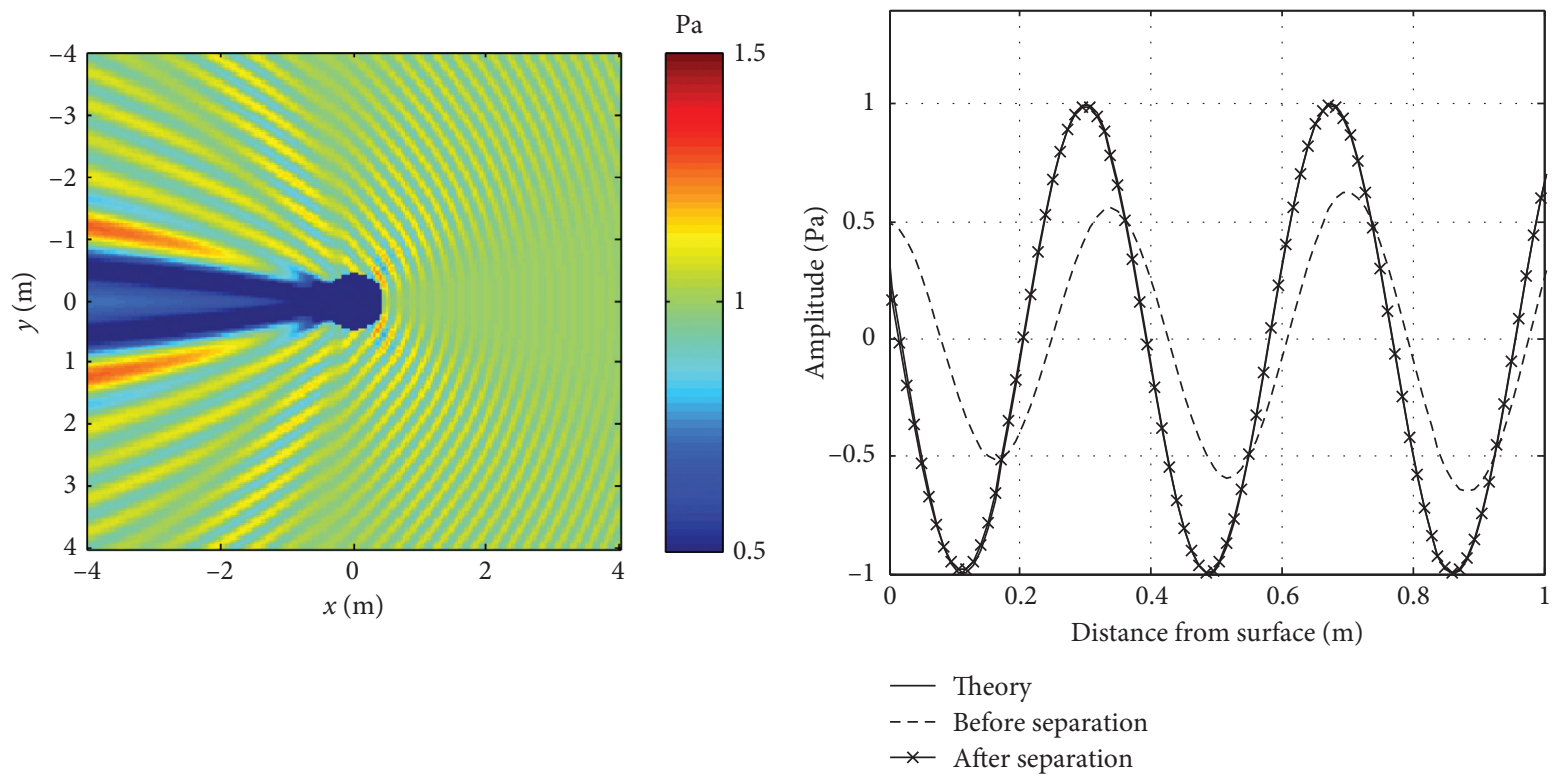

(a)

(b)

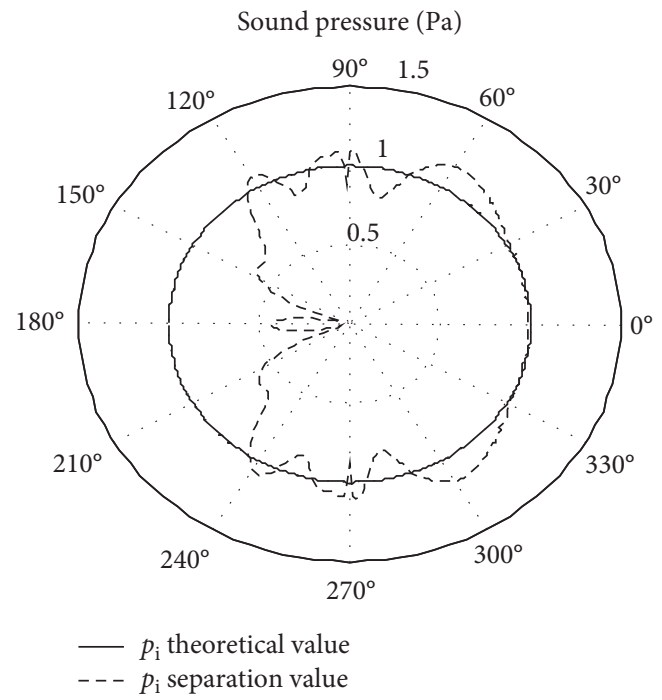

(c)

FIGURE 7: Separation results of the incident sound field on the surface of a cylinder. (a) Separated sound field. (b) Time-domain waveform. (c) Change in incident sound field at $0.75 \mathrm{~m}$ with azimuth.

point $\mathrm{A}$ or point $\mathrm{B}$ near the cylinder surface. When it was located at point $\mathrm{B}$, the receiver deviated from the $x$-axis, and the horizontal azimuth deviation was $\phi$.

Under the condition of plane wave incidence, the relationship between sound pressure and particle velocity is as follows:

$$
v=-\frac{1}{\rho} \int \frac{\partial p}{\partial t} \mathrm{~d} t=\frac{p}{\rho c} .
$$

Regardless of frequency, the wave impedance is $Z=p / v=\rho c$. Considering only the $x$-axis, assuming sound pressure $x(t)$, the relationship between vibration speed and sound pressure is given by

$$
\left\{\begin{array}{l}
p(t)=x(t) \\
v_{x}(t)=\frac{x(t)}{(\rho c)} \cdot \cos (\phi), \\
v_{y}(t)=\frac{x(t)}{(\rho c)} \cdot \sin (\phi)
\end{array}\right.
$$




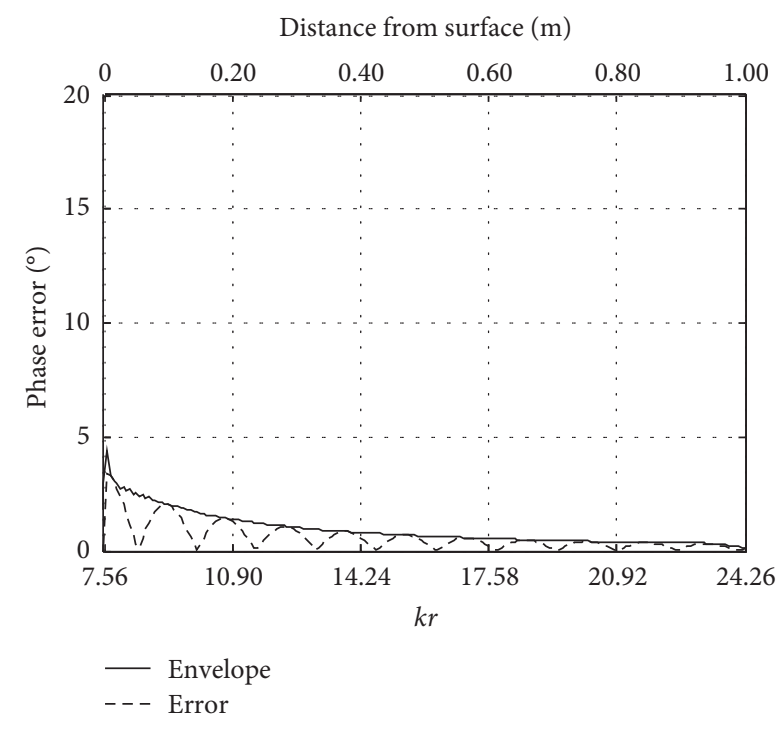

(a)

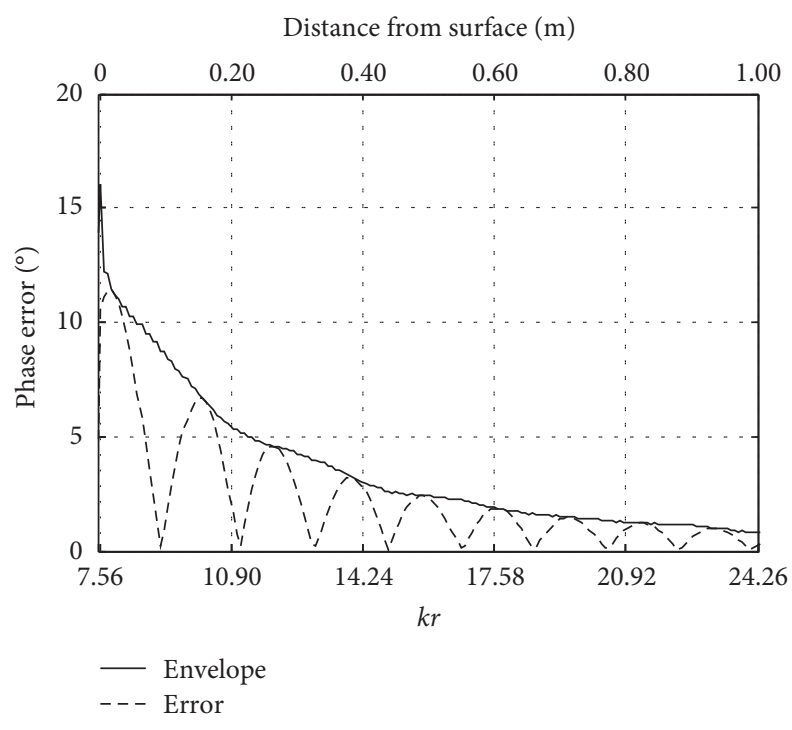

(b)

FIgURE 8: Variation of sound pressure phase separation error with distance in the incident sound field. (a) 0 degrees. (b) 30 degrees.

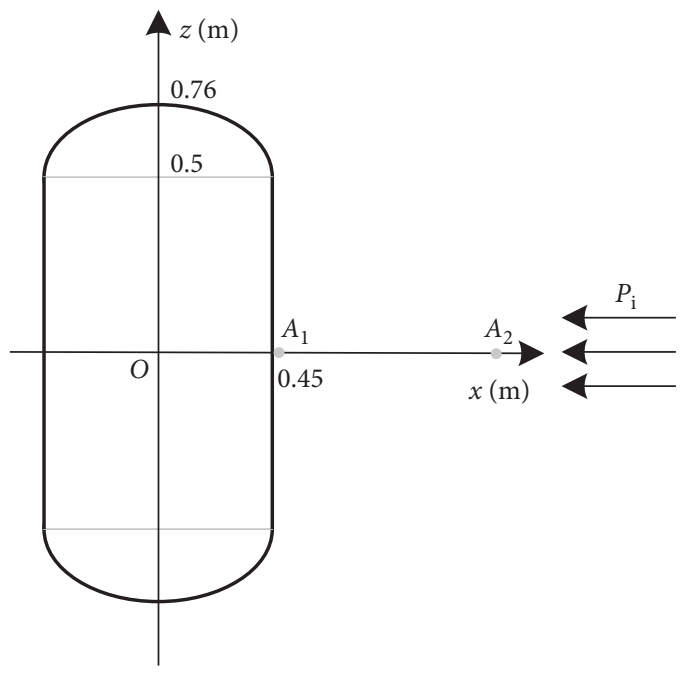

Figure 9: Model of a single-layer cylindrical shell.

According to the mathematical expression linking the scalar sound pressure and the vibration speed, the linear weighted joint processing was used. That is,

$$
p_{x}(t)=\frac{p(t)+\rho c v_{x}(t)}{2}=\frac{(1+\cos (\phi))}{2} x(t) .
$$

The vibration velocity was multiplied by the impedance $\rho c$. The physical unit is consistent with the sound pressure. The directivity of the joint processing is shown in Figure 6.

For the incident sound field, if $\phi_{i}=0$, then $p_{x}(t)=p_{i}(t)$. When the azimuth $\left(\phi_{i}\right)$ of the incident sound field is not the same as the direction $\left(v_{x}\right)$ of the vector sensor, it can be processed by $v_{x}$ and $v_{y}$. This means the vector beam was rotated in order to ensure that the direction of the unilateral cardioid was consistent with the direction of the incident sound field. By using this principle, plane waves in different directions can be obtained.

The sound field on the surface of a cylinder is the superposition of a far-field incident plane wave and a near-field scattered wave. The near-field scattered wave does not have the characteristics of a plane wave. The above equation (6) therefore becomes

$$
\begin{aligned}
p_{x}(t) & =\frac{\left(p_{\mathrm{i}}(t)+p_{s}(t)\right)+\rho c\left(v_{i x}(t)+v_{\mathrm{s} x}(t)\right)}{2} \\
& =\frac{\left[p_{\mathrm{i}}+\rho c v_{i x}(t)\right]}{2}+\frac{\left[p_{s}+\rho c v_{\mathrm{s} x}(t)\right]}{2} \\
& =p_{\mathrm{i}}(t)+\frac{\left[p_{\mathrm{s}}+\rho c v_{s x}(t)\right]}{2} .
\end{aligned}
$$

As the propagations of the incident and backscattered sound fields are in opposite directions, the joint treatment of sound pressure and vibration velocity forms a unilateral directivity. The direction of the maximum pressure is very small $\left(\right.$ near $\left.\phi_{s}=0\right)$ for the incoming direction of the incident sound, and the second term in equation (7) is related to the azimuth and distance. Although the scattered wave in the near-field does not have the characteristics of a plane wave, equation (7) is still meaningful. Based on this, the separation of the incident sound field under the condition of a complex sound field on the surface of a cylindrical target was analyzed.

\subsection{Results and Discussion: Separation Results of the Incident} Sound Field on the Surface of a Cylinder. Using equation (7), we obtained the incident sound field on an infinitely long cylindrical surface and analyzed its separation effect. Figure 7 shows the two-dimensional distribution of the incident sound field separation, the separation waveform at $0^{\circ}$ 


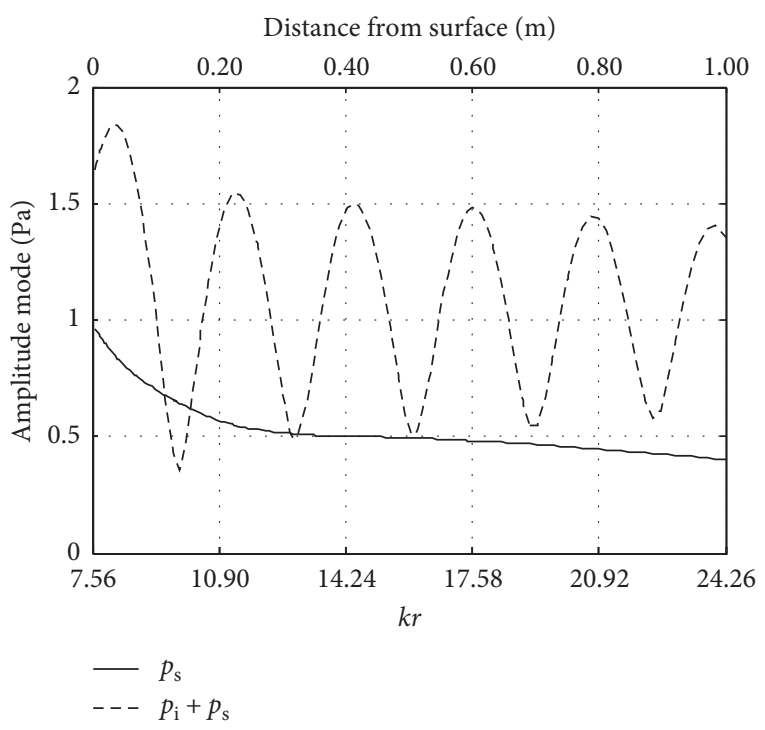

(a)

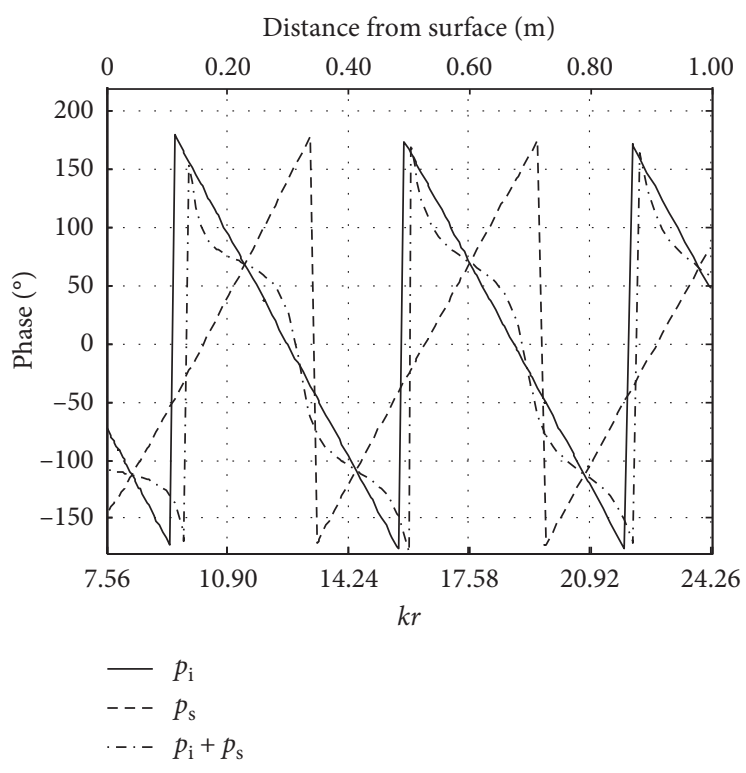

(b)

Figure 10: Distribution of sound pressure and phase with distance from the model. (a) Amplitude. (b) Phase.

azimuth (positive half-axis azimuth of the $\mathrm{X}$-axis, that is, the direction of the sound wave). For the relationship between the separation value of the incident sound field and the theoretical value (free field conditions), the typical distance is $0.75 \mathrm{~m}$, which is a function of the angle.

The main scattered wave in the range of $\pm 30^{\circ}$ around the $x$-axis is aligned with the direction of the smallest directivity, and the separation of the incident sound field is good. The smaller the angle, the higher the separation accuracy. The time-domain waveform of $0^{\circ}$ azimuth separation is shown in Figure 7(b). When the azimuth is greater than 30 degrees, the separation result becomes worse, as shown in Figure 7(c).

Due to the attenuation of the scattering field, the sound pressure amplitude and phase separation error (deviation between the separation value and the theoretical value) of the incident sound field will decrease with the distance from the cylindrical surface. The calculation results for the amplitude and phase error at the deviation angles of two typical receiver points are shown in Figures 6 and 8, respectively. As a result of the phase modulation of the scattering and incident sound fields, the amplitude of the separation error fluctuates under the attenuation trend and is almost zero when the phase is similar. It must be noted that the greater the phase difference, the greater the separation error. With the increase in the angle of deviation, the fluctuation of the amplitude error also increases. On the targeted surface, the maximum error of the $0^{\circ}$ azimuth position is nearly $0.5 \mathrm{~dB}$, while that of the $30^{\circ}$ azimuth position is $1.3 \mathrm{~dB}$. After $0.3 \mathrm{~m}$ from the target surface at $30^{\circ}$ azimuth, the amplitude error is less than $0.5 \mathrm{~dB}$.

The phase separation error shows a similar trend. For the $30^{\circ}$ azimuth deviation angle, the separation amplitude error is less than $5^{\circ}$ after $0.3 \mathrm{~m}$ from the targeted surface as shown in Figure 8.

\section{Model Verification and Analysis}

In order to further verify the vector separation method of the incident sound field, a physical model of a single-layer cylindrical shell with a spherical cap on both sides and a finite length was examined by numerical and sea tests.

3.1. Model Numerical Verification. The near-field scattering of a finite-length axisymmetric cylindrical shell can be obtained by converting the weak form volume fraction in a three-dimensional space into the area fraction on a twodimensional surface to obtain the scattered sound field [19].

The cylindrical shell model is shown in Figure 9. The geometric parameters include the following: outer radius $a=0.45 \mathrm{~m}$, shell thickness $h=0.01 \mathrm{~m}$, cylindrical shell length $l=1 \mathrm{~m}$, and crown height $=0.26 \mathrm{~m}$; material parameters include the following: $\rho=7850 \mathrm{~kg} / \mathrm{m}^{3}$, Poisson's coefficient $\sigma=0.28$, and elastic modulus $E=2.1 \times 10^{11} \mathrm{~N} / \mathrm{m}^{2}$; the density and sound velocity of the medium inside and outside are as follows: $\rho_{1}=1.29 \mathrm{~kg} / \mathrm{m}^{3}$ and $c_{1}=346 \mathrm{~m} / \mathrm{s}$, and $\rho_{2}=$ $1000 \mathrm{~kg} / \mathrm{m}^{3}$ and $c_{2}=1500 \mathrm{~m} / \mathrm{s}$.

Taking the geometric center of the cylindrical shell as the origin, the plane wave was incident along the negative $x$-axis direction, and the frequency was $4 \mathrm{kHz}$. The scattered sound pressure and vibration velocity in the horizontal direction (A1-A2 direction) of the shell surface were calculated. The sound pressure and vibration velocity of the incident sound field were given according to the steady-state signal of the plane wave, and the total sound field was obtained by equation (3).

Similar to the calculation results of infinitely long columns, the total sound pressure amplitude scattered from the surface of the finite cylindrical shell fluctuates with distance, and the phase value varies relative to the incident sound 


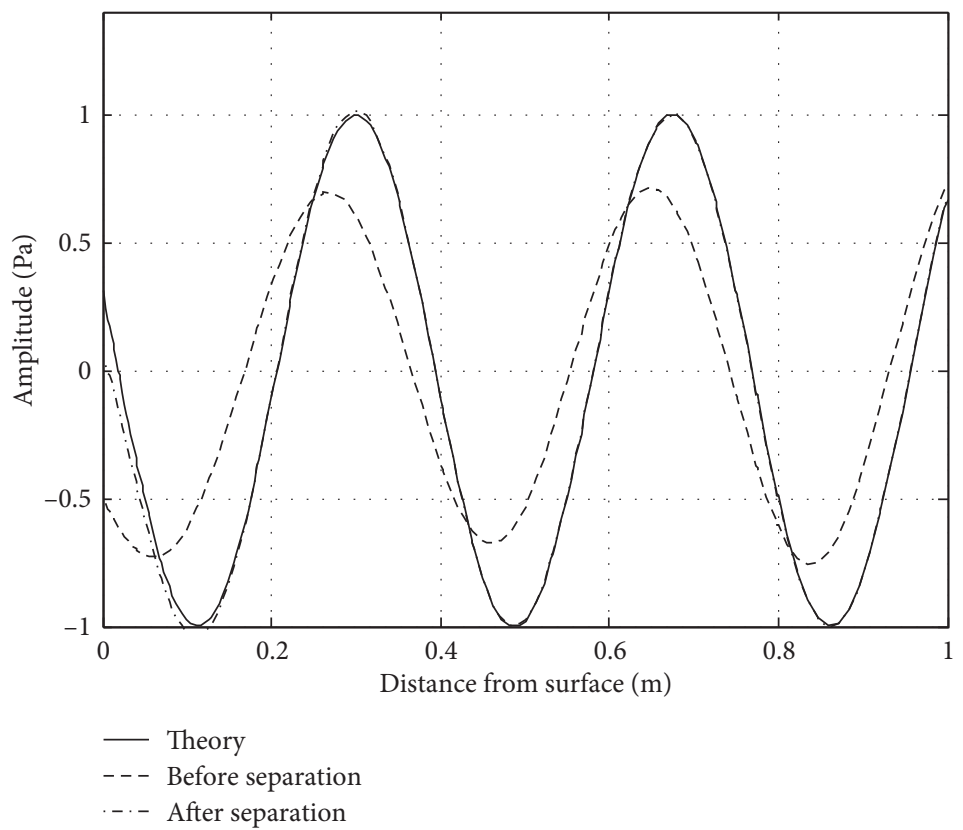

(a)

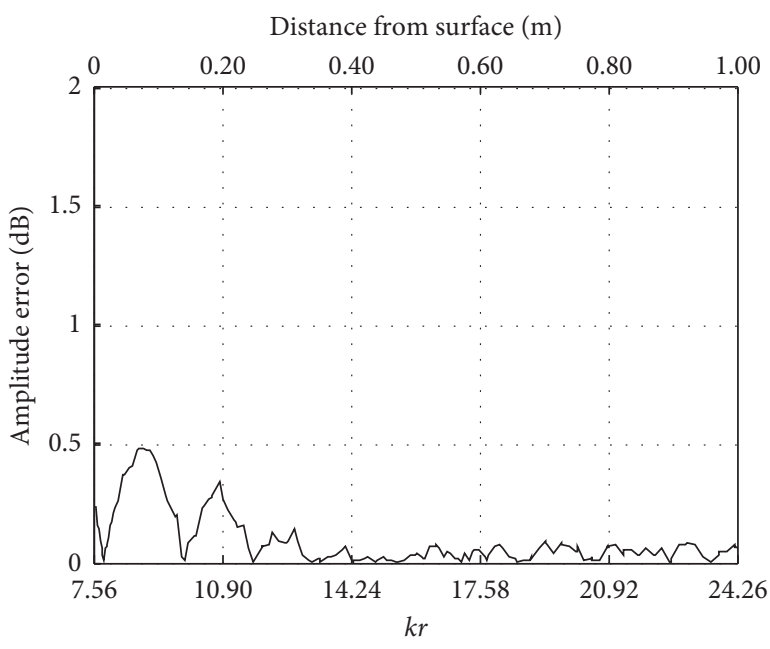

(b)

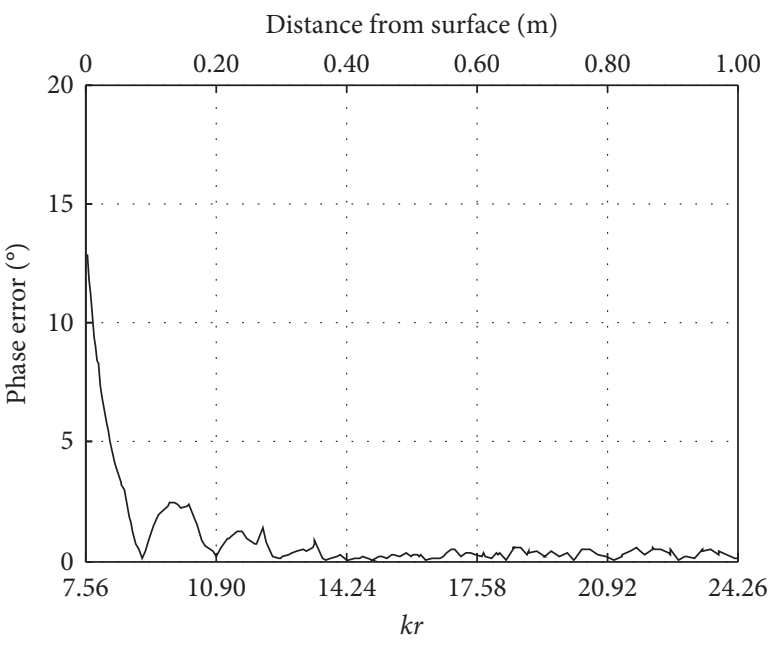

(c)

Figure 11: Variation of separation error of the incident sound field from the surface of the model. (a) Time-domain waveform. (b) Amplitude. (c) Phase.

field. As the distance from the cylinder increases, the amplitude and phase fluctuations decrease. At a distance of $0.6 \mathrm{~m}(k r=17.6)$ from the target surface, relative to the theoretical value of the incident sound field, the amplitude difference is still more than $50 \%$, and the phase difference is $20^{\circ}$ as shown in Figure 10. However, due to the elasticity of the shell, the initial phases of the incident sound field and scattered sound field from the targeted surface are no longer in phase, and the positions of the maximum and minimum values are also shifted.

Further analysis of the time-domain waveform and error of the incident sound field separation in the horizontal direction (A1-A2 direction) from the finite cylindrical shell surface are shown in Figure 11. The results are consistent with that of the analytical solution for the infinite cylinder and the errors in the amplitude and phase decrease with distance. Due to the finite calculation steps and overall modeling accuracy, the separation error curve is not as smooth as the analytical solution.

3.2. Model Test Verification. Based on the numerical analysis, the single-layer cylindrical shell was used as the target to perform the incident sound field separation test. The layout of the offshore test system is shown in Figure 12. The water depth in the sea area is $8 \mathrm{~m}$, and the test frequency is $4 \mathrm{kHz}$. The sound source and target were fixed below the lifting device of the twin-body measurement ship, located $4 \mathrm{~m}$ underwater, and meet the far-field conditions. A comparison between the sound source and the target was used for the 


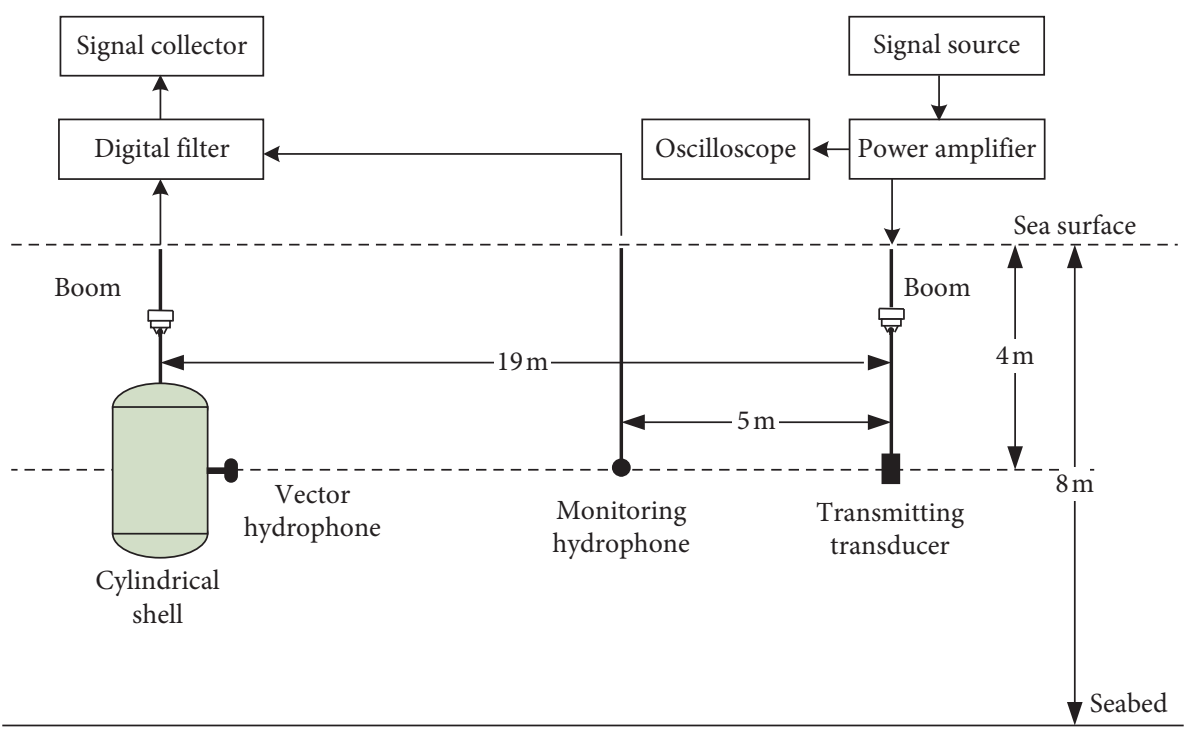

FIGURE 12: Seaborne layout of the incident sound field separation test.

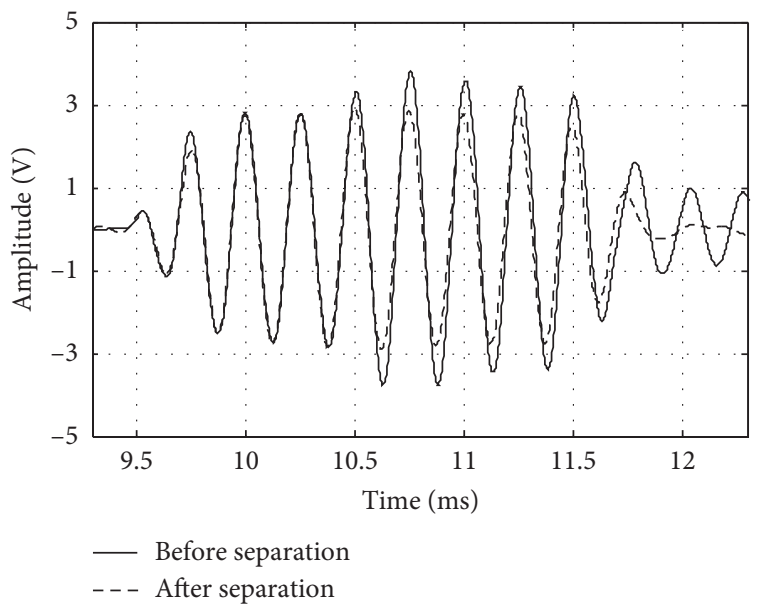

(a)

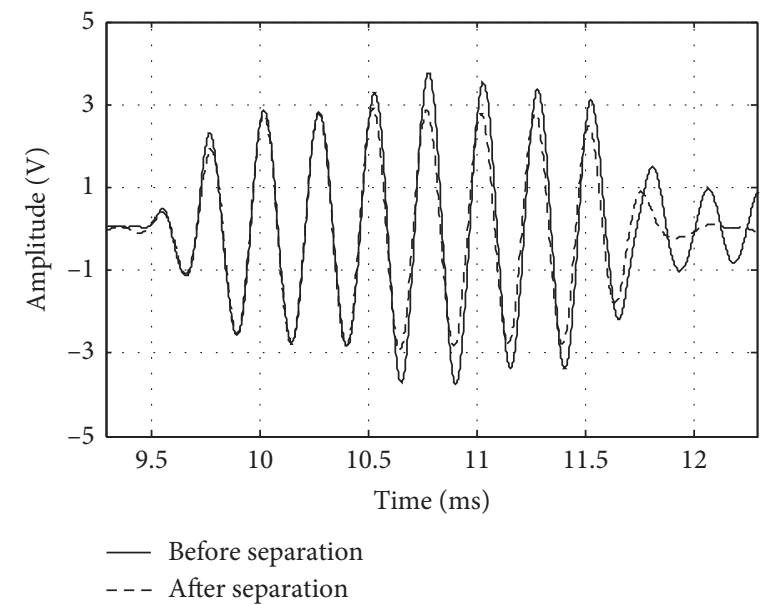

(b)

Figure 13: Time-domain signal of the incident sound field separation test. (a) $0^{\circ}$. (b) $30^{\circ}$.

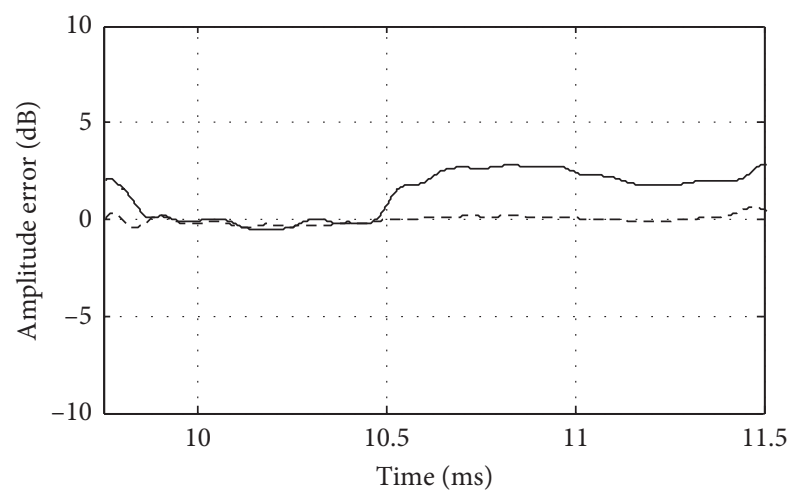

— Before separation

- - - After separation

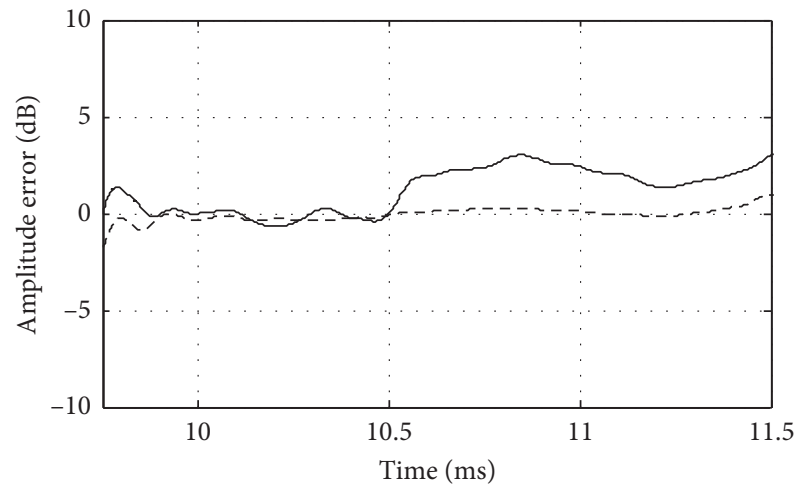

- Before separation

- - - After separation

(a)

(b)

FIgURE 14: Amplitude error of the incident sound field separation test. (a) $0^{\circ}$. (b) $30^{\circ}$. 


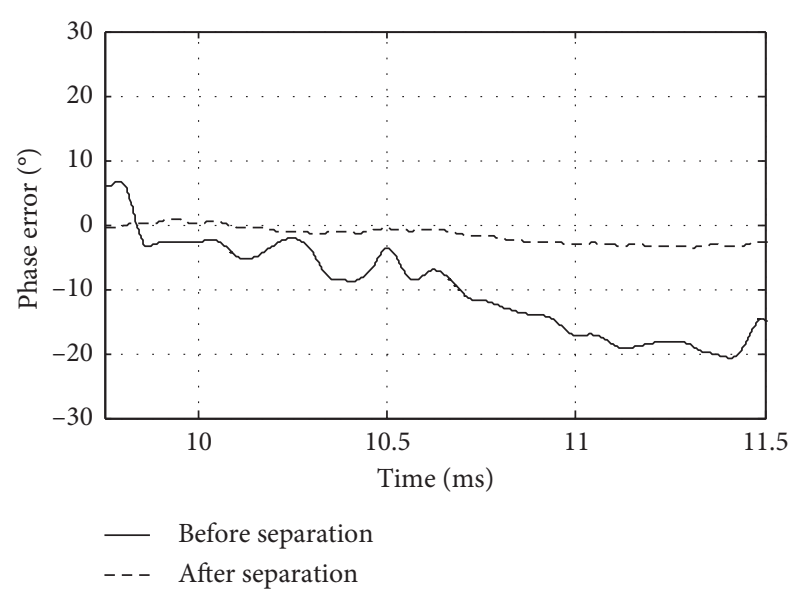

(a)

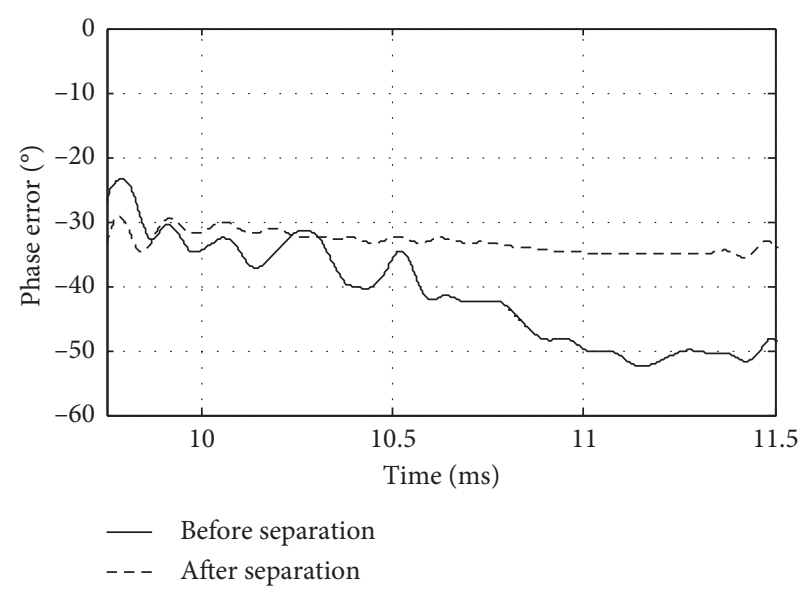

(b)

Figure 15: Phase error of the incident sound field separation test. (a) $0^{\circ}$. (b) $30^{\circ}$.

comparative analysis of the sound field. According to the previous analysis, the two test conditions of $0^{\circ}$ and $30^{\circ}$ azimuth deviation of the receiving point were considered.

In the test, in order to reduce the influence of other scatterings, the pulse width of the transmitted signal was reduced to $2 \mathrm{~ms}$. Compared with the monitoring signal, affected by the near-field scattering of the target surface near the receiving point, the sound-field signal received by the test vector sensor is significantly superimposed.

Figure 13 shows the time-domain signal of the sound field separation test on the surface of a cylindrical shell. Because the vector sensor received the incident wave before it was scattered from the cylindrical shell, it shows a pure incident wave in the first $0.4 \mathrm{~ms}$ (approximately 2 signal periods); thereafter, the target scattered wave arrives at the sensor, and the signal amplitude varies significantly. After the vector separation process, the signal contaminated by the shell scattering basically reached the level of the first two cycles. Affected by the target scattering, the amplitude of the incident wave sound pressure varies by $3-5 \mathrm{~dB}$, and the maximum phase variation reaches $35^{\circ}$ as shown in Figures 14 and 15. After the vector processing, the deviation of the sound pressure amplitude is less than $1 \mathrm{~dB}$ for the two receiver positions (the overall deviation of the $0^{\circ}$ position is slightly less than that of the $30^{\circ}$ position). The phase deviation is also greatly reduced. The phase deviation is less than $3^{\circ}$ at the $0^{\circ}$ position and less than $5^{\circ}$ at the $30^{\circ}$ position. This is consistent with the calculation results.

The results of the numerical and sea tests show that the combined treatment of sound pressure and vibration velocity can effectively suppress the scattering interference of the cylindrical target surface. High-precision information of the amplitude and phase of the sound pressure in the incident sound field was obtained by separation.

\section{Conclusion}

This paper presents a vector processing method for the separation of the incident sound field after scattering from the surface of a cylindrical shell and further analyzes the influence of the received sound between the double-layer cylinder and the scattering of the sensor itself. The theoretical analysis and experimental verification show that the linear combined processing of scalar sound pressure and vector vibration velocity can effectively suppress the scattered sound field from the cylindrical surface and, hence, separate and obtain high-precision incident sound field pressure amplitude and phase information. The separation effect in front of the cylinder ( $0^{\circ}$ direction) is better compared to off-axis positions. As the distance from the cylindrical surface increases, the amplitude and phase separation deviation of the incident sound field gradually decreases. At a typical distance acceptable for practical applications, the amplitude deviation of the measured incident sound pressure is less than $1 \mathrm{~dB}$, and the phase deviation is less than $3^{\circ}$. It has certain engineering application value in underwater acoustic measurement, navigation, and so on.

\section{Data Availability}

The data used to support the findings of this study are available from the corresponding author upon request.

\section{Conflicts of Interest}

The authors declare that there are no conflicts of interest regarding the publication of this paper.

\section{Acknowledgments}

This project was funded by the Ship Fund Project (7131304).

\section{References}

[1] Z. Y. He, "Review of some aspects of underwater noise including its control techniques and its prospect," Applied Acoustics, vol. 21, no. 2, pp. 26-34, 2002.

[2] J. P. Carneal and C. R. Fuller, "An analytical and experimental investigation of active structural acoustic control of noise 
transmission though doubles panel system," Journal of Sound Vibration, vol. 272, no. 3-5, pp. 749-771, 2004.

[3] E. G. Thomas and J. S. Joseoh, "A model for sonar interrogation of complex bottom and surface targets in shallow-water waveguides," Journal of the Acoustical Society of America, vol. 123, no. 4, pp. 2024-2034, 2008.

[4] B. Zhang, X. G. Zhang, and Z. C. Ma, "Discussion on the physical significance of the acoustic target strength of an underwater object in shallow water and modal-filtering measurement," Journal of Ship Mechanics, vol. 19, no. 3, pp. 322-326, 2015.

[5] R. A. Kosobrodov and V. N. Nekrasov, "Effect of the diffraction of sound by the carrier of hydroacoustie equipment on the results of measurements," Acoustical Physics, vol. 47, no. 3, pp. 382-388, 2001.

[6] G. L. Liang, F. B. Pang, and G. P. Zhang, "The effect of sound scattering by elastic spherical shell on the direction finding accuracy of vector sensors," Journal Vibration and Shock, vol. 33, no. 3, pp. 46-50, 2014.

[7] M. T. Cheng, J. A. Mann, and A. Pate, "Wave-number domain separation of the incident and scattered sound field in Cartesian and cylindrical coordinates," The Journal of the Acoustical Society of America, vol. 97, no. 4, pp. 2293-2303, 1995.

[8] K. SaijYou, M. Ueda, and W. Youshikawa, "Application of generalized near-field acoustical holography to scattering problem," Japanese Journal of Applied Physics, vol. 33, pp. 3120-3135, 1991

[9] Z. Y. He, "Inverse problem of acoustics: technology of acoustic holographic transformation and discrimination of source characteristics," Acta Physica Sinica, vol. 16, no. 3-4, pp. 600-612, 1996.

[10] X. M. Bao and Z. Y. He, "Research on holographic reconstruction of target scattering field," Acta Acoustic, vol. 25, no. 3, pp. 254-264, 2000.

[11] F. Yu, J. Chen, W. B. Li, and X. Z. Chen Xinzhao, "Sound field separation technology and its application in near-field acoustic holography," Acta Physica Sinica, vol. 54, no. 2, pp. 699-706, 2005.

[12] Y. B. Zhang, L. Xu, C. X. Bi, and X. Z. Chen, "Single-surface sound field separation technology based on sound pressurevibration velocity measurement," Acta Physica Sinica, vol. 58, no. 12, pp. 8364-8371, 2009.

[13] W. B. Li, M. Z. Lian, C. X. Bi, J. Chen, and X. Z. Chen, "The theory of separation of incident and scattered sound in spherical coordinate system," Science China Technology Sciences, vol. 37, no. 1, pp. 99-106, 2007.

[14] R. A. Kosobrodov and V. N. Nekrasov, "Effect of the diffraction of sound by the carrier of hydroacoustic equipment on the results of measurement," Acoustical Physics, vol. 47, no. 3, pp. 382-388, 2001.

[15] J. Y. Hui, H. Liu, H. B. Yu, and M. Y. Fan, "Probe into the joint information processing and physical basis of acoustic pressure and velocity," Acta Acoustic, vol. 25, no. 4, pp. 303-307, 2000.

[16] J. Y. Hui, C. X. Li, G. L. Liang, and H. Liu, "Combined signal processing of sound pressure and vibration velocity against coherent interference," Acta Acoustic, vol. 26, no. 5, pp. 389-394, 2000.

[17] X. H. Chen, P. Cai, J. Y. Hui, and G. L. Liang, "Directivity of acoustic vector array," Acta Acoustic, vol. 28, no. 2, pp. 142-143, 2003.

[18] D. S. Yang, Z. R. Zhu, and S. G. Shi, "Vector characteristics of near-field sound scattering from a spherical baffle," Journal Vibration and Shock, vol. 32, no. 2, pp. 135-139, 2013.
[19] D. Lu, "Sound scattering calculation of elastic target based on finite element principle," M.S. thesis, Harbin Engineering University, Harbin, China, 2014 